\title{
Correction: Mahmood et al. Hard Real-Time Task Scheduling in Cloud Computing Using an Adaptive Genetic Algorithm. Computers 2017, 6, 15
}

\author{
Amjad Mahmood ${ }^{1}$, Salman A. Khan ${ }^{2, *}$ and Rashed A. Bahlool ${ }^{3}$ \\ 1 Computer Science Department, University of Bahrain, P.O. Box 32038, Sakhir, Bahrain; \\ amjadmahmud@gmail.com \\ 2 Computer Engineering Department, University of Bahrain, P.O. Box 32038, Sakhir, Bahrain \\ 3 College of Information Technology, University of Bahrain, P.O. Box 32038, Sakhir, Bahrain; \\ rashed.b63@gmail.com \\ * Correspondence: sakhan@uob.edu.bh; Tel.: +973-1743-7673
}

Received: 13 June 2018; Accepted: 13 June 2018; Published: 15 June 2018

In article by Mahmood [1], the results for a genetic algorithm (GA), adaptive genetic algorithm (AGA), and greedy algorithm were not correctly reported in Section 5 due to a programming error. The authors have now repeated the experiments with extensive simulations and analysis using the help of an assistant (Mr. Rashed A. Bahlool), who has been added as an author. Note that, since the core focus of the paper is on the performance of an adaptive genetic algorithm and greedy algorithm, the central claims and conclusions have not been affected, but the tables and figures have been updated with correct values, and necessary changes to the text have been made, as explained below.

1. Table 4 is updated with corrected results as follows. Furthermore, results for 150 and 250 tasks have been added for a better understanding of the trends for each combination.

Table 4. Average cost for the four combinations. The best results are highlighted in bold.

\begin{tabular}{ccccc}
\hline No. of Tasks & $\begin{array}{c}\text { Combination 1 } \\
\text { Average Cost }\end{array}$ & $\begin{array}{c}\text { Combination 2 } \\
\text { Average Cost }\end{array}$ & $\begin{array}{c}\text { Combination 3 } \\
\text { Average Cost }\end{array}$ & $\begin{array}{c}\text { Combination 4 } \\
\text { Average Cost }\end{array}$ \\
\hline 10 & $\mathbf{1 5 2 . 9 2} \pm \mathbf{2 . 9 8}$ & $176.44 \pm 3.42$ & $191.07 \pm 3.26$ & $156.69 \pm 3.03$ \\
20 & $327.74 \pm 4.35$ & $336.57 \pm 4.69$ & $346.27 \pm 4.83$ & $\mathbf{3 2 4 . 9 6} \pm \mathbf{4 . 3 2}$ \\
30 & $403.36 \pm 4.92$ & $399.81 \pm 5.01$ & $431.04 \pm 5.03$ & $\mathbf{3 8 1 . 4 3} \pm \mathbf{5 . 1 4}$ \\
40 & $753.56 \pm 6.25$ & $756.76 \pm 6.13$ & $\mathbf{7 3 4 . 5 9} \pm \mathbf{5 . 9 6}$ & $746.92 \pm 5.89$ \\
60 & $1114.66 \pm 7.23$ & $\mathbf{1 0 8 8 . 5 6} \pm \mathbf{7 . 1 7}$ & $1123.06 \pm 6.93$ & $1090.37 \pm 7.07$ \\
80 & $1393.10 \pm 9.12$ & $1401.43 \pm 9.08$ & $1445.16 \pm 8.83$ & $\mathbf{1 3 8 7 . 8 8} \pm \mathbf{9 . 1 3}$ \\
100 & $1776.54 \pm 11.72$ & $1672.05 \pm 10.84$ & $1691.36 \pm 10.21$ & $\mathbf{1 6 6 3 . 9 9} \pm \mathbf{1 1 . 3 6}$ \\
150 & $2356.24 \pm 10.23$ & $\mathbf{2 3 1 2 . 9 5} \pm \mathbf{9 . 9 2}$ & $2402.09 \pm 10.73$ & $2387.36 \pm 11.15$ \\
200 & $2659.68 \pm 11.69$ & $2728.16 \pm 12.32$ & $2749.42 \pm 12.11$ & $\mathbf{2 6 2 6 . 5 2} \pm \mathbf{1 1 . 8 9}$ \\
250 & $3582.56 \pm 13.71$ & $\mathbf{3 5 3 3 . 2 6} \pm \mathbf{1 2 . 5 3}$ & $3613.21 \pm 12.66$ & $3552.67 \pm 14.83$ \\
300 & $4098.61 \pm 13.63$ & $\mathbf{3 9 4 7 . 8 1} \pm \mathbf{1 4 . 5 6}$ & $4105.31 \pm 13.95$ & $4052.33 \pm 14.36$ \\
\hline
\end{tabular}

2. Page 13, the paragraph below Table 3 has some changes in the text. This paragraph describes the observations in updated Table 4 in point (1) above. The overall claim that Combination 4 is the best remains unaffected, but the relative performance of the four combination types has changed.

Table 4 provides the results for the four combinations for each test case. It is observed from these tables that for the majority of test cases, Combination 4 produced the best results. More specifically, Combination 4 generated the minimum cost for 20, 30, 80, 100, and 200 tasks. Combination 2 produced 
the best results for four test cases, which were 60,150, 250, and 300. The other two combinations produced the best results for only one test case each, making them the worst performers. Therefore, based on the results, it can be fairly claimed that Combination 4 was much better than combinations 1 and 2, while showing relatively better performance than Combination 3 as far as the quality of results is concerned.

3. Figure 7 has been updated with corrected runtimes. Also, runtimes for 150 and 250 tasks have been added.

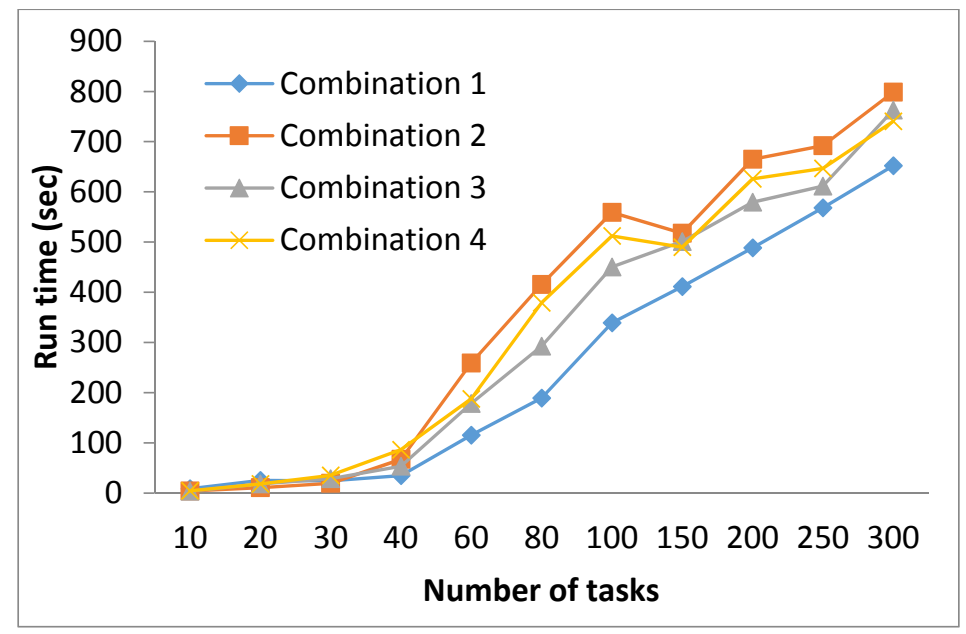

Figure 7. Comparison of execution times for the four combinations.

4. Table 6 has been updated with the corrected costs for a GA, AGA, and greedy algorithms. Also, costs for 150 and 250 tasks have been added.

Table 6. Cost comparison of genetic algorithm $(G A)$, adaptive genetic algorithm $(A G A)$, and greedy algorithms.

\begin{tabular}{cccc}
\hline No. of Tasks & Greedy & GA & AGA \\
\hline 10 & 198.23 & 152.92 & 146.65 \\
20 & 358.59 & 324.96 & 319.26 \\
30 & 417.11 & 381.43 & 362.47 \\
40 & 898.79 & 734.59 & 718.44 \\
60 & 1232.35 & 1088.56 & 1046.31 \\
80 & 1524.85 & 1387.88 & 1332.21 \\
100 & 1896.32 & 1663.99 & 1551.68 \\
150 & 2621.58 & 2312.95 & 2171.58 \\
200 & 2889.73 & 2626.52 & 2438.52 \\
250 & 4117.54 & 3533.26 & 3258.65 \\
300 & 4426.36 & 4052.33 & 3813.08 \\
\hline
\end{tabular}

5. Figure 8 has been updated according to the results given in Table 6 (point (4) above). 


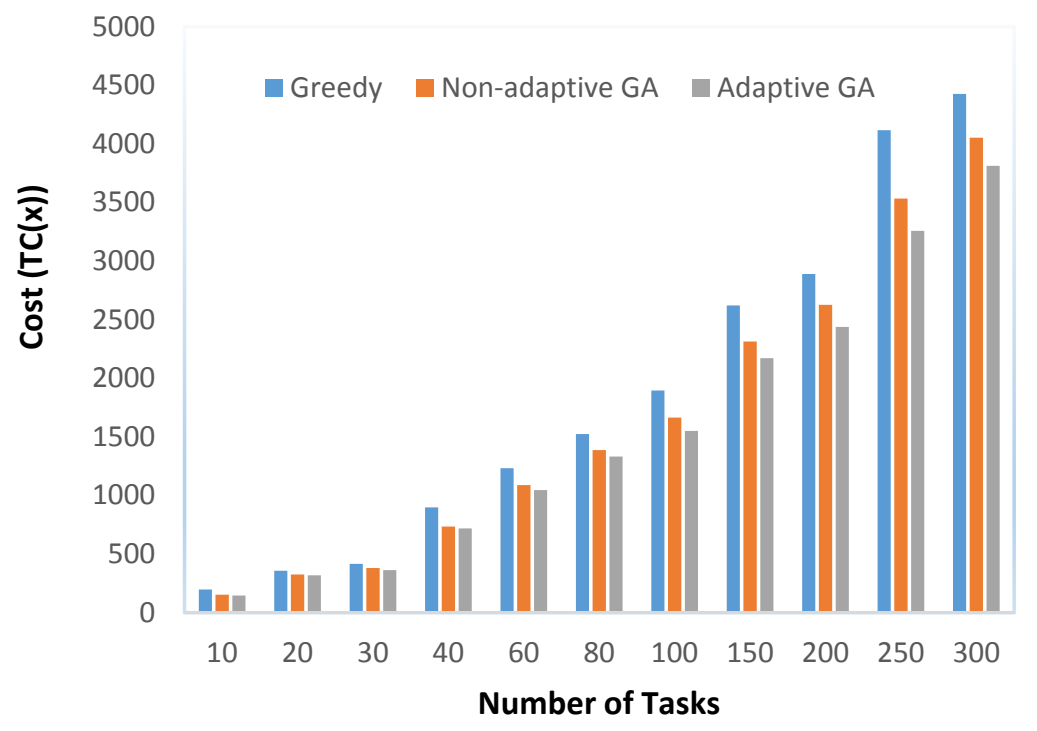

Figure 8. Comparison of cost for Greedy, GA, and AGA.

6. The percentage improvements in Table 7 have been updated according to the corrected results given in Table 6 (given in point (4) above).

Table 7. Percentage improvement obtained by Adaptive GA with respect to Greedy, and GA.

\begin{tabular}{ccc}
\hline Tasks & Adaptive GA vs. Greedy & Adaptive GA vs. GA \\
\hline 10 & 35.17 & 4.28 \\
20 & 12.32 & 1.79 \\
30 & 15.07 & 5.23 \\
40 & 25.10 & 2.25 \\
60 & 17.78 & 4.04 \\
80 & 14.46 & 4.18 \\
100 & 22.21 & 7.24 \\
150 & 20.72 & 6.51 \\
200 & 18.50 & 7.71 \\
250 & 26.36 & 8.43 \\
300 & 16.08 & 6.27 \\
\hline
\end{tabular}

7. In Section 5.3, Second paragraph, the last five lines have been re-written as follows:

The results indicate that the improvements achieved by an AGA with respect to the greedy algorithm were significantly high, in the range of $12 \%$ to over $35 \%$. As far as a GA and AGA are concerned, the percentage improvements by an AGA were in the range of over $1.79 \%$ to $8.43 \%$. These improvements were subjected to statistical testing. The results of this statistical testing indicated that a huge majority of improvements achieved by an AGA were statistically significant. Two exceptions were test cases with 20 and 40 tasks where the improvements were not statistically significant.

8. Figure 9 has been updated as below. 


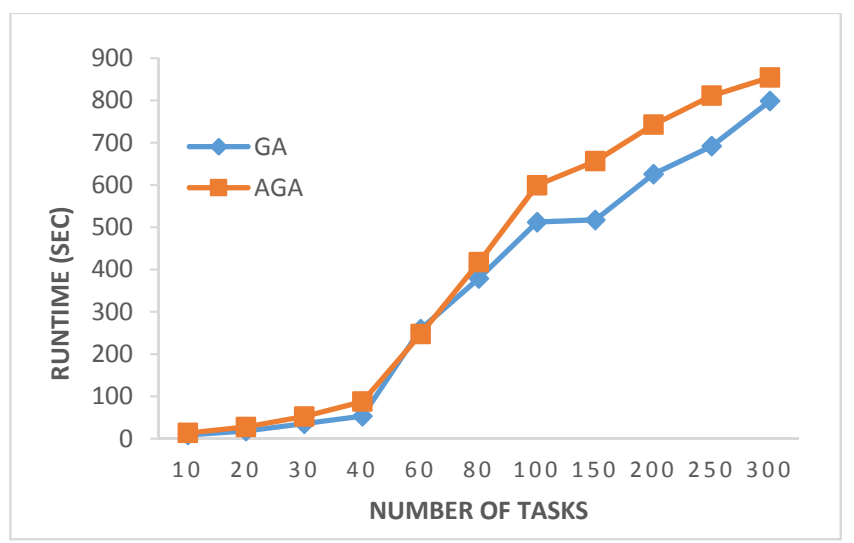

Figure 9. Run time comparison of a GA and AGA.

9. In the last paragraph of Section 5.3, the last eight lines of the paragraph have been re-written as follows:

The figure indicates that an AGA maintained sufficient diversity until the end of execution, while a GA with four combinations was unable to maintain diversity for longer durations. More specifically, for combinations 1 and 3, the diversity almost became zero before 330 iterations. Combinations 2 and 4 were better than the other two combinations in the sense that the diversity was maintained until around 400 iterations. However, for an AGA, the population had non-zero diversity, even at the end of execution, as is evident from Figure 10e. Therefore, it can be confidently claimed that an AGA was better than the other four combinations in terms of maintaining population diversity and consequently giving solutions of better quality.

10. Plots in Figure 10 have been regenerated according to the new simulations leading to corrected results. The updated Figure 10 is as given below.

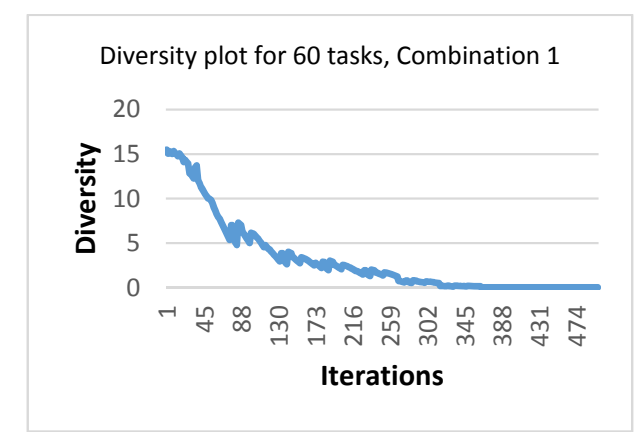

(a)

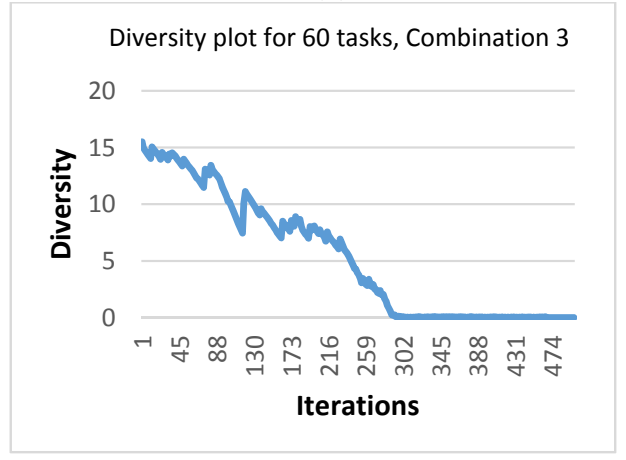

(c)

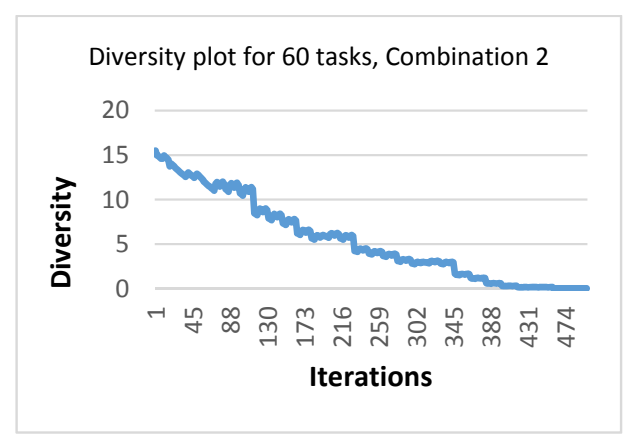

(b)

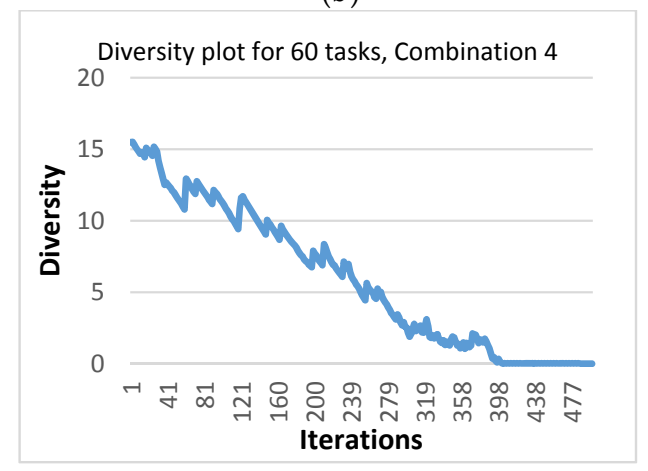

(d)

Figure 10. Cont. 


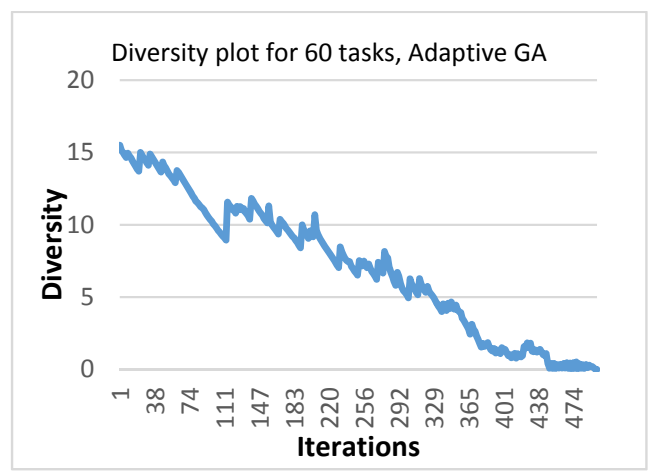

(e)

Figure 10. Diversity plots for the typical runs for 60 tasks using (a) Combination 1; (b) Combination 2; (c) Combination 3; (d) Combination 4; and (e) AGA.

11. Figure 1 has been re-drawn with a new structure without changing any information in the Figure. The updated figure is as follows.

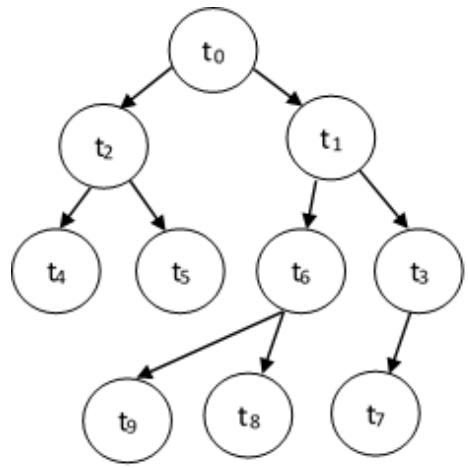

Figure 1. A DAG with 10 tasks.

12. The first line of Section 4.1 has the term (GA) removed. The updated line is:

Greedy algorithms have been extensively studied.

13. The first line of Section 4.2 has the term (GA) added. The updated line is:

The genetic algorithm (GA) is one of the most well-known evolutionary algorithms that is based on the concept of natural evolution [40].

The authors apologize for any inconvenience caused.

Author Contributions: A.M. worked on the theoretical development of the proposed AGA and the greedy algorithm and contributed to writing the relevant portions of the manuscript. S.A.K. and R.A.B. performed the experiments, analyzed the results and contributed in writing the relevant portions of the manuscript. All authors have read and approved the final manuscript.

\section{Reference}

1. Mahmood, A.; Khan, S.A.; Bahlool, R.A. Hard Real-time Task Scheduling in Cloud Computing using an Adaptive Genetic Algorithm. Computers 2017, 6, 15. [CrossRef]

(C) 2018 by the authors. Licensee MDPI, Basel, Switzerland. This article is an open access article distributed under the terms and conditions of the Creative Commons Attribution (CC BY) license (http://creativecommons.org/licenses/by/4.0/). 